\title{
Review of: "The interplay between sulfur metabolism and desulfurization profile in Rhodococcus: Unraveling the role of the transsulfuration pathway"
}

Emmanuel Duval

Potential competing interests: The author(s) declared that no potential competing interests exist.

\section{Comment 1/}

Line 25 "Several natural biocatalysts have been isolated, all harboring the highly conserved desulfurization operon dszABC."

Some isolates possess the 45 phenotype while it was proved that they do not harbor any dsz operon, nor relatives (Duval, 2020; Narayanan, 2020; Wang et al., 2015)

\section{Comment 2/}

Line 446 "Growth took place in 96-well cell culture plates (F-bottom; Greiner Bio-One, Fischer Scientific, US) with $200 \mu \mathrm{L}$ working volume in thermostated plate-shakers at $30^{\circ} \mathrm{C}$ and $600 \mathrm{rpm}$ (PST-60HL, BioSan, Pegasus Analytical SA, Hellas). For each condition, an initial biomass concentration of 0.045-0.055 g/L was applied, while 20 identical well-cultures were used."

How was filled up the plate? How was maintained axenic condition during incubation (cap, lid, etc.)? How was avoided evaporation on the plate egde ? For this last, I guess outer wells were filled up with water, bringing the number of working wells to $96-[2 *(8+12-2)]=60$ per plate.

\section{Comment 3/}

Line 392 "For growth tests on solid media, R. qingshengii cells were grown on basal salts medium (BSM) prepared according to (Karimi et al., 2017), containing $0.165 \mathrm{M}$ ethanol (0.33 M carbon) as carbon source and $1 \% \mathrm{w} / \mathrm{v}$ agarose.

Line 779 “Figure S1 (B) Growth test of wild-type (wt) R. qingshengii IGTS8 and knockout strains cbs $\Delta$, metB $\Delta . "$

$\lfloor!$ IMPORTANT $\lfloor! \backslash$ Was the negative control for growth (without any sulfur source) tested ? Agar purity was not specified, it could contain sulphur residues that may support strain growth without any other sulphur source added in the medium (see the provider website).

\section{Comment 4/}

Lines 731, 738, 743, 749: Titles of figures 5, 6, 7 and 8.

Line 232: "To determine the effect of [...] deletion on [...] desulfurization activity in the presence of sulfate, 
methionine or cysteine as sole sulfur sources"

Those titles suggest that DBT desulfurization occurred meanwhile other sulphur sources were added to the medium. For example, Figure 6: "Recombinant strains desulfurize in the presence of sulfate."

Considering Materials and Methods, desulfurization activity was carried on with resting cells grown with other sulphur sources than DBT (sulfate, DMSO, Met or Cys), and suspended in HEPES buffer medium with DBT, am I right?

In general, all along the article, there is a doubt about the desulfurization/BDS phenotype occurrence in the presence or not of other sulphur sources alongside DBT. At the end, it is confusing because I wonder if the aim (and result) was to obtain mutants able to desulfurize DBT meanwhile other sulphur sources are present in the medium.

\section{Comment 5/}

Lines 227: "was increased", "compared", etc.

!I IMPORTANT $\lfloor\backslash$ A rigorous comparison of quantitative results, and moreover its interpretation, must be conducted with a statistical test to determine whether the difference between two means is significant or not.

\section{Comment 6/}

Line 286

I guess a verb is missing in the sentence.

\section{Comment 7/}

Line 355: "sulfate [...] did not seem to be directly involved in the repression system"

Just to discuss, this statement contrasts with the results displayed with the strain Gordonia sp. IITR100 where dsz molecular regulation was partly elucidated showing the involvement of a TerT-like protein. This protein binds DNA at the dsz operon promoter sequence, and and can also bind sulfate and DBT, acting as a repressor in the first case and as a inducer in the second (Murarka and Srivastava, 2019; Murarka et al., 2019).

Thus, dsz regulation may differ from a strain or genus to another, considering regulatory sequences upstream the $d s z$ operon are not conserved between BDS ${ }^{+}$strains.

\section{CITATIONS}

Duval, E. (2020). Biotransformation microbienne optimisée des sulfones issues des fiouls marins. Université de Pau et des Pays de l'Adour.

Murarka, P., and Srivastava, P. (2019). Characterization of DNA binding and ligand binding properties of the 
TetR family protein involved in regulation of dsz operon in Gordonia sp. IITR100. Int. J. Biol. Macromol. 141, 671-679.

Murarka, P., Bagga, T., Singh, P., Rangra, S., and Srivastava, P. (2019). Isolation and identification of a TetR family protein that regulates the biodesulfurization operon. AMB Express 9, 71.

Narayanan, S.M.A. (2020). Investigation of new actinobacteria for the biodesulphurisation of diesel fuel. Napier University.

Wang, J., Davaadelger, B., Salazar, J.K., Butler, R.R., Pombert, J.-F., Kilbane, J.J., and Stark, B.C. (2015). Isolation and characterization of an interactive culture of two Paenibacillus species with moderately thermophilic desulfurization ability. Biotechnol. Lett. 37, 2201-2211. 